LAWRENCE LIVERMORE NATIONAL LABORATORY

\title{
Effects of the Electron Energy Distribution Function on Line and Continuum Emission
}

S. B. Hansen and A. S. Shlyaptseva

\section{$4 / 15 / 2004$}

Atomic Processes in Plasmas, Santa Fe, NM, April 19-22, 2004 
This document was prepared as an account of work sponsored by an agency of the United States Government. Neither the United States Government nor the University of California nor any of their employees, makes any warranty, express or implied, or assumes any legal liability or responsibility for the accuracy, completeness, or usefulness of any information, apparatus, product, or process disclosed, or represents that its use would not infringe privately owned rights. Reference herein to any specific commercial product, process, or service by trade name, trademark, manufacturer, or otherwise, does not necessarily constitute or imply its endorsement, recommendation, or favoring by the United States Government or the University of California. The views and opinions of authors expressed herein do not necessarily state or reflect those of the United States Government or the University of California, and shall not be used for advertising or product endorsement purposes. 


\title{
Effects of the Electron Energy Distribution Function on Line and Continuum Emission
}

\author{
Stephanie B. Hansen*\$ and Alla S. Shlyaptseva* \\ * Department of Physics/220, University of Nevada, Reno, Nevada, 89557 \\ ${ }^{\S}$ Lawrence Livermore National Laboratory, PO Box 808, Livermore, CA 94551
}

\begin{abstract}
Effects of suprathermal ("hot") electrons on the predictions of K- and L-shell nonLTE collisional-radiative atomic kinetics models are presented through an investigation of various electron distribution functions (EDFs) on collisional rates and spectra. It is shown that while most collisional rates are fairly insensitive to the functional form and characteristic energy of the hot electrons as long as their characteristic energy is larger than the threshold energy for the collisional process, collisional excitation and ionization rates are highly sensitive to the fraction of hot electrons. This permits the development of robust spectroscopic diagnostics that can be used to detect the presence of hot electrons from $\mathrm{x}$-ray line emission spectra. Hot electrons are shown to increase and spread out plasma charge state distributions, amplify the intensities of emission lines fed by direct collisional excitation and radiative cascades, and alter the structure of satellite and EUV line emission features. The characteristic energy, functional form, and spatial properties of hot electron distributions in plasmas are open to characterization through their effects on high-energy continuum emission and on the polarization of spectral lines.
\end{abstract}

\section{INTRODUCTION}

Suprathermal ("hot") electrons are of significant interest in studies of a variety of plasma sources, including laser-produced plasmas [1-4], pulsed-power plasmas [5-7], and astrophysical sources [8,9]. Hot electrons are typically generated in such sources by strong electric fields (including resonant laser-plasma interactions) and can have significant influence on plasma radiative properties and dynamic development.

The effects of hot electrons on bulk plasma properties can be deleterious (e.g. target preheat in laser-driven inertial confinement fusion schemes [4]) or beneficial (e.g. increased high-energy radiation from relatively low-power plasma sources [1-3, 5-7]). In both cases, reliable diagnostics to detect their presence and measure the properties of hot electrons are the first step towards understanding and controlling their effects.

Collisional-radiative (CR) models have been used extensively over the last few years to study the effects of hot electrons on K-shell line emission [1, 5, 6, 10]. More recently, the effects of hot electrons on L-shell line emission from pulsed power [7] and laser plasma sources $[2,3]$ have been investigated. The models are used to generate synthetic $\mathrm{x}$-ray emission spectra that can be compared with experimental data to diagnose the conditions of the experimental source.

In this paper, the effects of hot electrons on x-ray line and continuum emission from $\mathrm{Ti}$ and $\mathrm{Kr}$ are investigated. The intent is to clarify the diagnostic information 
present in spectral regions with photon energies from EUV to many keV to inform experimental measurements and to maximize the information gleaned from the plasma emission. To that end, a discussion of the sensitivity of various plasma materials to small fractions of hot electrons is given after the description of hot electron effects on various spectral regions.

\section{MODELED ELECTRON DISTRIBUTION FUNCTIONS AND THEIR EFFECTS ON COLLISIONAL RATES}

Hot electrons affect the predictions of collisional-radiative models through their influence on modeled collisional rates, which are typically obtained by integration of collision cross sections over a two-component electron distribution function that varies with the electron energy $\varepsilon$ :

$$
F(\varepsilon)=(1-f) F_{M}\left(\varepsilon ; T_{e}\right)+f F_{H}\left(\varepsilon ; \varepsilon_{H}\right)
$$

In (1), $F_{M}$ is a Maxwellian distribution at a bulk electron temperature $T_{e}, F_{H}$ is a hot electron distribution with characteristic energy $\varepsilon_{H}$, and $f$ is the fraction of hot electrons to the total electron density. Figure 1 shows three functional forms (Gaussian, powerlaw, and Maxwellian) that we have used to model hot electron distributions. Combinations of these forms can reproduce a wide range of non-equilibrium EDF behavior. These distributions are discussed in detail in [11].

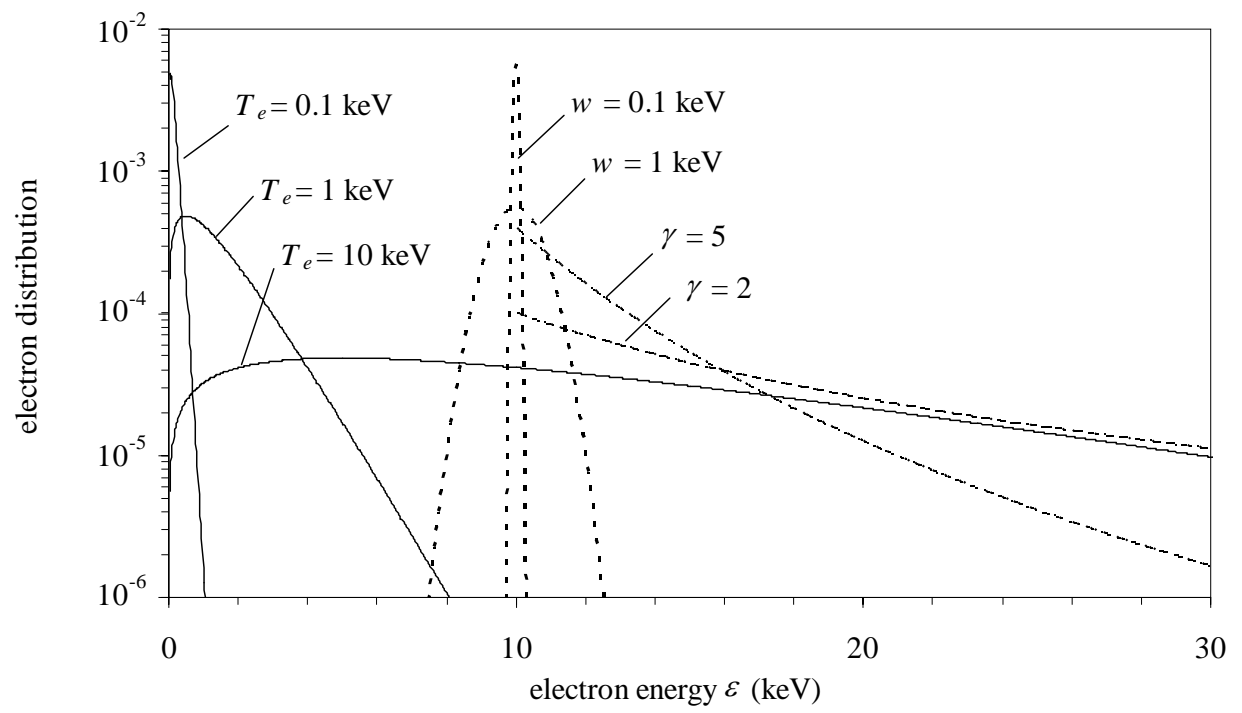

FIGURE 1. Sample hot electron distribution functions: Maxwellian (solid lines) at various electron temperatures $T_{e}$, Gaussian (dotted lines) centered at characteristic energy $\varepsilon_{H}=10 \mathrm{keV}$ with various halfwidths at half-max given by $w \sqrt{ } \ln 2$, and power-law (dashed lines) with characteristic energy $\varepsilon_{H}=10$ $\mathrm{keV}$ and various decay constants $\gamma$.

Although a wide range of characteristic energies and functional forms of the hot electron distribution are commonly used (chosen to reflect properties of hot electron characteristics in a variety of plasmas), it turns out that many important collisional 
rates are fairly insensitive to the functional form of the electron distribution and $\varepsilon_{H}$ as long as $\varepsilon_{H}$ is larger than the transition energy $\Delta E$ of the collisional processes. This is illustrated for resonance collisional excitation and collisional ionization in Fig. 2, which shows the results of integrating collision cross sections that decay as $\ln (\varepsilon) / \varepsilon$ over a variety of EDFs. Because the cross sections vary relatively slowly with the impact electron energy $\varepsilon$, the exact distribution of electrons with sufficient energy to cause the collisional process is relatively unimportant. However, collisional excitation and ionization rates depend very strongly on the fraction of electrons with $\varepsilon>\Delta E$ and can increase by orders of magnitude when small fractions of hot electrons are added to cool bulk Maxwellian distributions. By contrast, most collisional recombination and de-excitation processes are only slightly affected by small fractions of hot electrons, since those processes are dominated by lower-energy electrons in the bulk Maxwellian distribution. De-excitation and recombination processes are therefore fairly insensitive to the precise functional form and energy of the hot electron distribution. Details of the integration and the EDF dependence of non-resonant collisional excitation, deexcitation, and three-body and radiative recombination processes are given in [11].

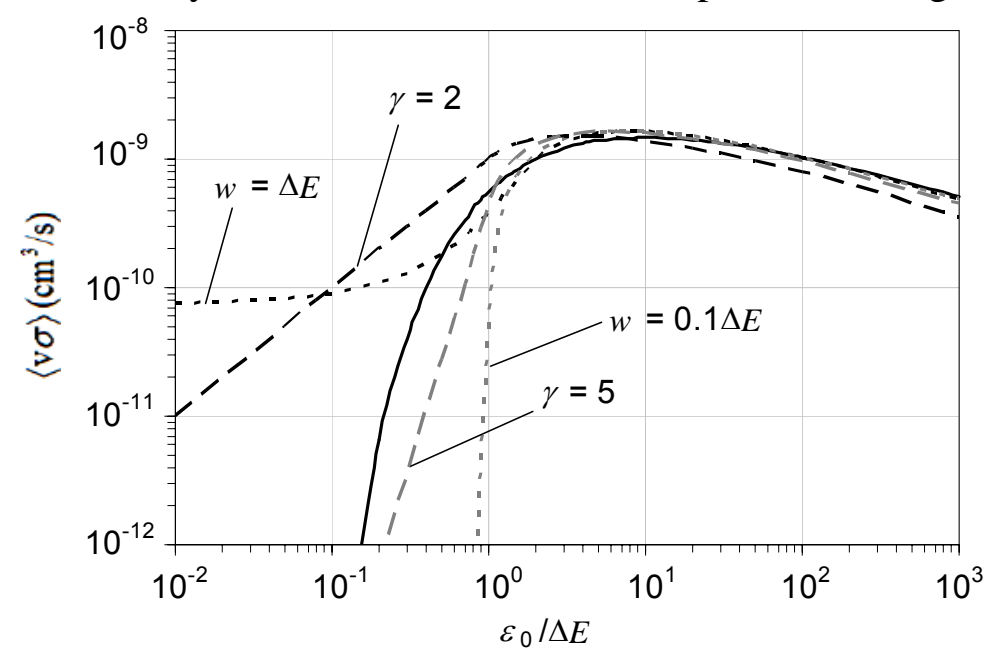

FIGURE 2. Rate coefficients for resonant collisional excitation or collisional ionization obtained by integrating a collision cross section proportional to $\ln (\varepsilon) / \varepsilon$ over Maxwellian (solid lines), Gaussian (dotted lines), and power-law (dashed lines) distributions. For $\varepsilon_{H} / \Delta E>1$, the value of $\varepsilon_{H}$ and the functional form of the electron distribution have a relatively minor influence on the collisional rates.

\section{HOT ELECTRON EFFECTS ON K- AND L-SHELL LINE EMISSION}

In the following sections, calculations from two collisional-radiative models are presented. The Ti model is based on data from the FAC atomic structure code [12] and includes the ground states of all ions, singly excited levels up to $n=5$ for $\mathrm{H}$ - and $\mathrm{H}$ like ions, $n=4$ for Li-like ions, and $n=3$ for Be-through Ne-like ions, and doubly excited levels up to $n=3$ for He-, Li-, and Ne-like ions and up to $n=2$ for Bethrough F-like ions. The L-shell Kr model is based on a compiled data set described fully in [2] and includes fine-structure levels for F- through Mg-like Kr. All levels are coupled within each ion by radiative decay and collisional excitation and de-excitation 
and are coupled between neighboring ions with collisional ionization, Auger decay, and their reverse rates as well as by radiative recombination.

Because including hot electrons in the plasma electron distribution increases the probability of direct collisional excitation and ionization processes without significantly affecting de-excitation and recombination, hot electrons tend to increase the average charge state of modeled plasma ions. In addition, they spread out the charge state balance, leading to the presence of emission from a wider range of ions than would be seen from a single-temperature plasma. This effect is illustrated in Fig. 3, which shows K-shell Ti spectra at conditions chosen to produce emission from Kshell resonance and satellite lines from He- to F-like Ti. All three spectra with hot electrons have smaller bulk electron temperatures and emission from a wider range of charge states than spectra without hot electrons at roughly equivalent average ion charges.

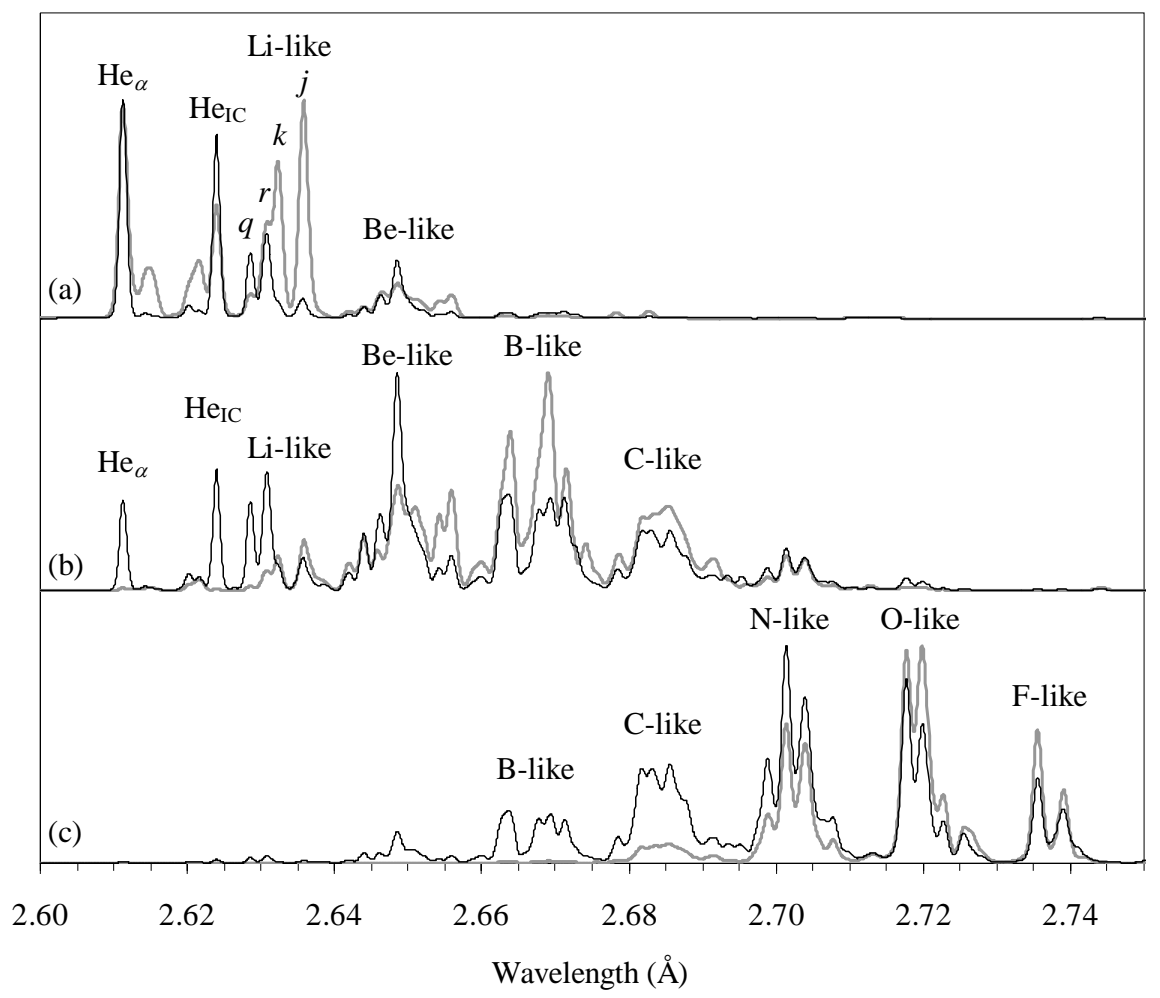

FIGURE 3. Effects of hot electrons on K-shell Ti spectra with $n_{e}=10^{20} \mathrm{~cm}^{-3}$ and various electron distributions. Gray lines: $f=0$ and $T_{e}=$ (a) 800, (b) 350, and (c) $200 \mathrm{eV}$. Black lines: $T_{e}=100 \mathrm{eV}$ and hot electrons in a Gaussian distribution with $\varepsilon_{H}=10 \mathrm{keV}, w=100 \mathrm{eV}$, and $f=$ (a) $10 \%$, (b) $3 \%$, and (c) $1 \%$.

Hot electrons also affect line emission from single charge states. First, they can significantly enhance the populations of excited levels and thereby increase the intensity of all line emission, particularly of high-energy lines. Second, they can change the shape of satellite spectra by amplifying collisionally excited inner-shell satellite lines relative to satellite lines populated by dielectronic recombination. Finally, by increasing the total population in excited levels, hot electrons can amplify the intensities of lines fed by radiative cascades, especially in closed-shell ions with 
relatively few dipole decay channels to the ground state. The latter two effects are illustrated by the spectra in Fig. 3. Higher-energy satellite lines (e.g. the Li-like lines labeled $q$ and $r$ ) are formed primarily by inner-shell collisional excitation and are significantly amplified by the presence of hot electrons, whereas lower-energy lines (e.g. the Li-like lines labeled $j$ and $k$ ) are formed through dielectronic recombination and are less sensitive to the presence of hot electrons. Figure 3 shows that hot electrons can also have a marked effect on the intensity of the He-like intercombination line $\left(\mathrm{He}_{\mathrm{IC}}\right)$. At low electron densities, $\mathrm{He}_{\mathrm{IC}}$ is fed by radiative cascades from highly excited He-like levels that cannot decay directly to the ground state. Hot electrons increase the total excitation into excited levels and thereby increase the number of radiative cascades, leading to a higher $\mathrm{He}_{\mathrm{IC}} / \mathrm{He}_{\alpha}$ ratio than would be expected at a given plasma density without hot electrons. Because in the absence of hot electrons, intense intercombination lines imply low electron densities and intense inner-shell satellite lines imply high electron densities, the simultaneous presence of both features in experimental spectra are strong evidence for the presence of hot electrons [6].

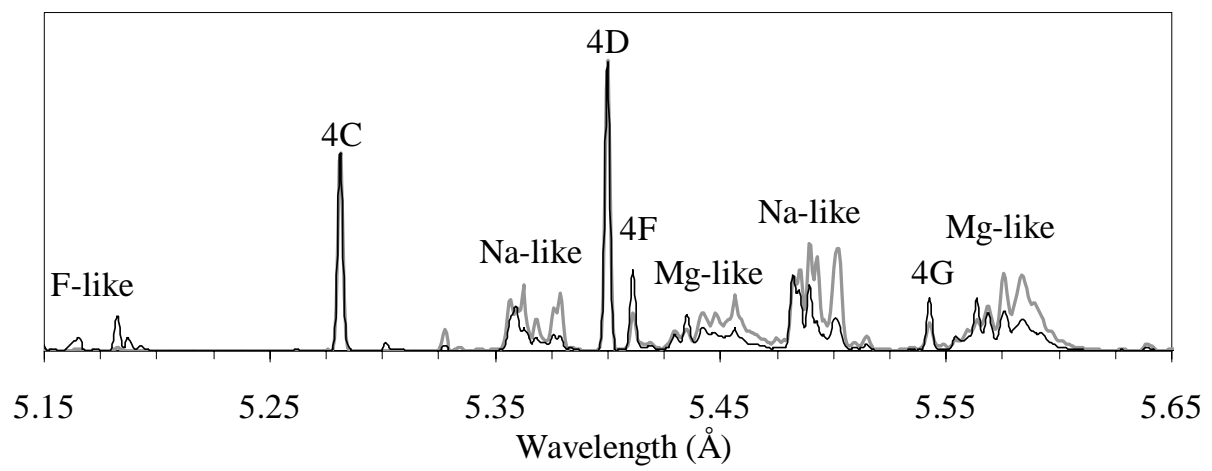

FIGURE 4. Effects of hot electrons on L-shell (4-2) Kr spectra with $n_{e}=10^{20} \mathrm{~cm}^{-3}$. The gray line shows spectra with $T_{e}=550 \mathrm{eV}$ and $f=0$ and the black line shows spectra with $T_{e}=250 \mathrm{eV}$ and $f=5 \%$ of hot electrons in a Gaussian distribution with $\varepsilon_{H}=20 \mathrm{keV}$ and $w=100 \mathrm{eV}$.

Figure 4 shows effects in L-shell $\mathrm{Kr}$ spectra similar to those described for K-shell Ti. Including hot electrons permits line emission from a wider range of charge states and amplifies the emission from cascade-fed Ne-like 4-2 resonance lines (4F and 4G) and the high-energy lines within Na- and Mg-like $\mathrm{Kr}$ satellite features.

\section{He-like Ti Line Polarization}

In addition to affecting the relative intensity of line emission through purely kinetic processes, hot electrons in anisotropic distributions (beams) can polarize line emission from transitions formed by collisional processes along the beam axis. By measuring parallel and perpendicular components of plasma emission simultaneously, the anisotropy of a hot electron distribution can be determined and limits can be imposed on the energies of the beam electrons.

Figure 5 shows how the polarizations of He-like Ti resonance and intercombination lines are affected by the energy of the impact electron. Near the threshold energies of the two transitions, $\mathrm{He}_{\alpha}$ is positively polarized and $\mathrm{He}_{\mathrm{IC}}$ is negatively polarized. Given 
a beam of such electrons, a spectrometer measuring parallel-polarized line emission would record a smaller $\mathrm{He}_{I C} / \mathrm{He}_{\alpha}$ ratio than one measuring perpendicularly polarized or unpolarized emission [13]. Since the relative polarization of $\mathrm{He}_{\alpha}$ and $\mathrm{He}_{\text {IC }}$ change with the impact electron energy, polarization measurements can in principle give information about the energy distribution of anisotropic electrons [15]. Of course, since the electron distribution also has a purely kinetic effect on line intensities, polarization effects must be convolved with population effects.

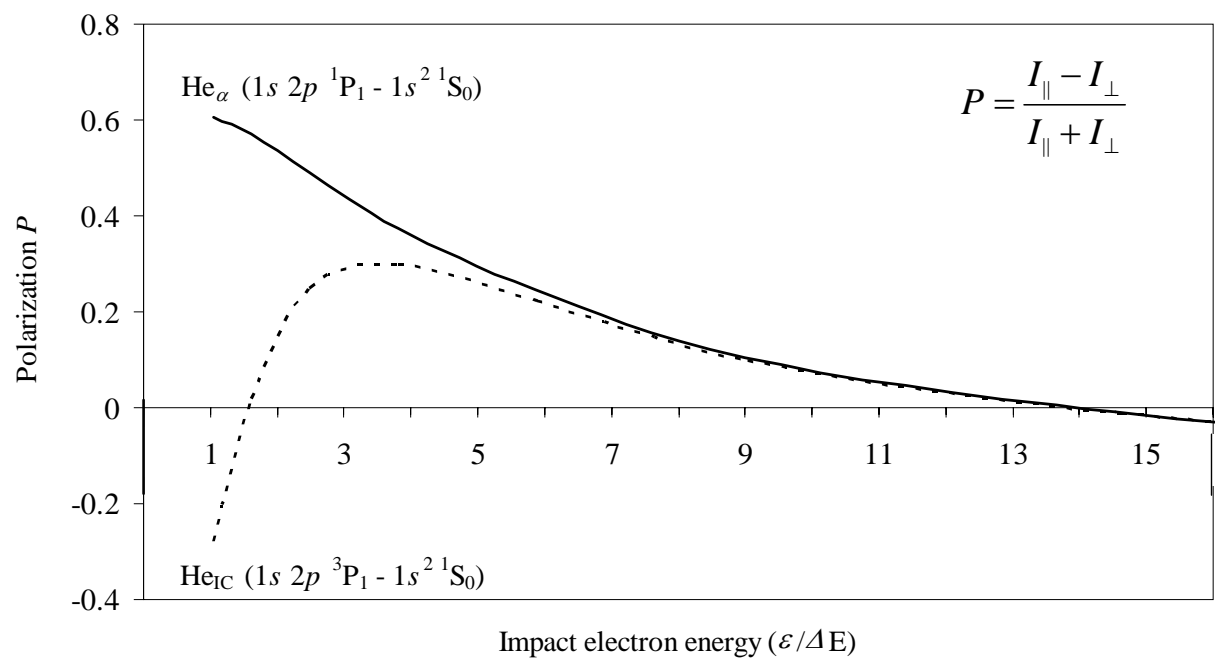

FIGURE 5. Theoretical polarizations of $\mathrm{Ti} \mathrm{He}_{\alpha}$ (solid line) and $\mathrm{He}_{\mathrm{IC}}$ (dashed line).

\section{BROADBAND EMISSION SPECTRA}

The signatures of hot electrons on charge state balances and line emission spectra discussed above are due primarily to the effects of hot electrons on collisional boundbound (excitation) and bound-free (ionization) rates. However, hot electrons also affect the free-free and free-bound processes that give rise to continuum radiation. Free-free bremsstrahlung radiation in particular can be used as an unambiguous indicator of the presence of hot electrons in plasma. In the present model, radiative recombination emission intensity is calculated using steady-state level populations and the same radiative recombination cross sections as are used in the CR calculations. The bremsstrahlung emission intensity is determined using free-free Gaunt factors approximated by a weighted average of the Kramers and Born values for Maxwellian distributions and the Born-Elwert formulation for non-Maxwellian distributions.

Figure 6 gives modeled broadband emission spectra from Ti with and without hot electrons. In both panels of the figure, bremsstrahlung emission is given by the dashed lines underlying the total emission, which includes line, radiative recombination, and bremsstrahlung emission. In addition to increasing and broadening the K-shell line emission features (which are detailed in Fig. 1), hot electrons significantly affect both lower-energy L-, M-, and inter-shell emission features, radiative recombination features, and high-energy continuum emission. 

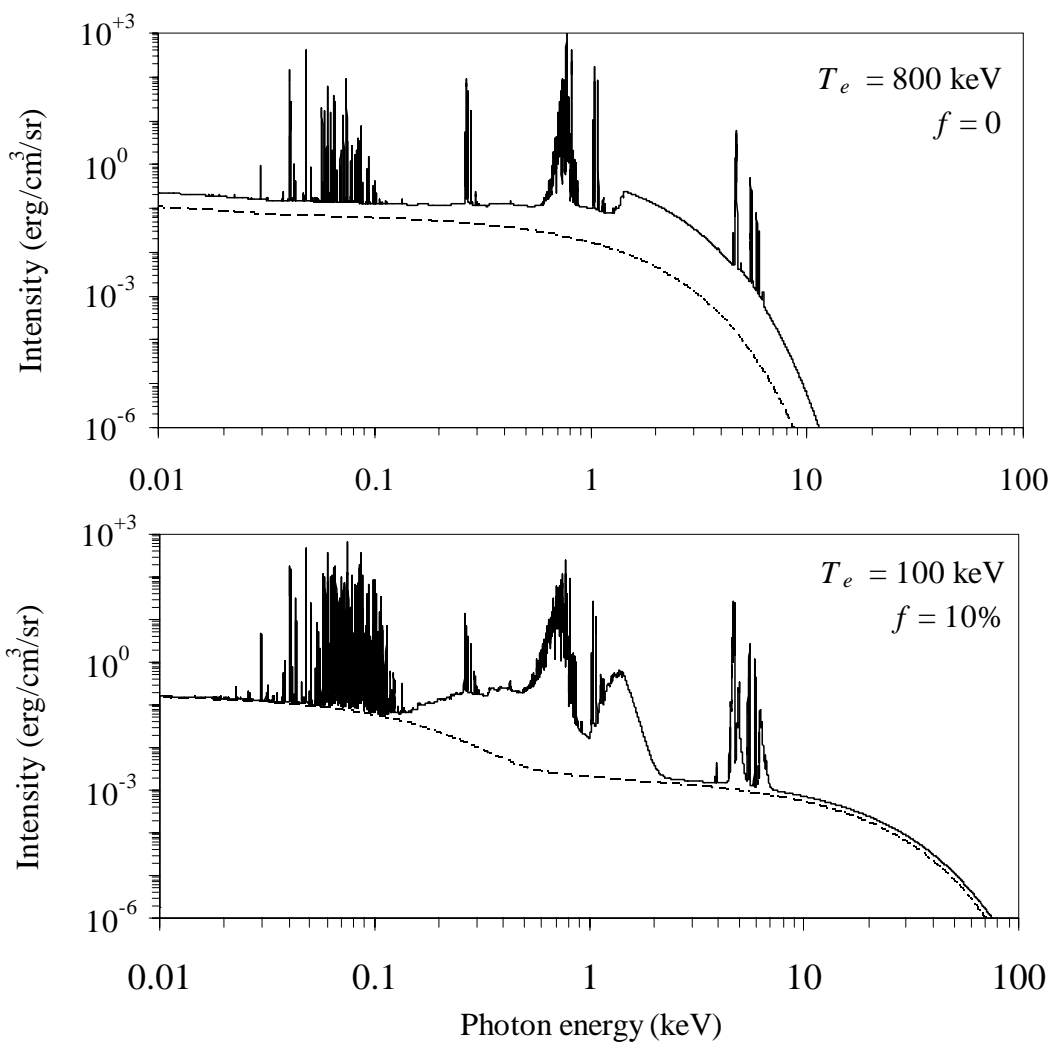

FIGURE 6. Effects of hot electrons on broadband Ti spectra with $n_{e}=10^{20} \mathrm{~cm}^{-3}$ with and without hot electrons (as labeled). The hot electrons in the bottom spectrum are in a Maxwellian distribution with $\varepsilon_{H}$ $=10 \mathrm{keV}$. Bremsstrahlung emission is given by the dashed lines in each spectrum.

\section{EUV Line Emission}

The L- and M-shell emission features of Ti are apparent in Fig. 6 near 1 and 0.3 $\mathrm{keV}$, respectively, and inter-shell transitions are present around $0.1 \mathrm{keV}$. The structures of these three line emission features are significantly changed when hot electrons are included in the CR calculations. The difference is due both to the spreading out of the charge state balance to include a larger number of ions and to the increased populations of highly excited levels within each ion. These effects tend to amplify and broaden the emission features between and among levels in excited configurations. This effect should be particularly apparent in low-density plasmas, in which ladder ionization cannot generate significant population in highly excited levels.

\section{Radiative Recombination Features}

In $\mathrm{Ti}$, radiative recombination processes to the $\mathrm{Li}$ - and Be-like ions have energies near $1.4 \mathrm{keV}$. In the top spectrum in Fig. 6, this recombination edge is fairly well defined and includes mostly recombination emission from transitions from the ground states of He- and Li-like Ti to Li- and Be-like Ti. When a significant fraction of hot electrons are included, as in the bottom spectrum of Fig. 6, the recombination feature is amplified and significantly broadened. The amplification is primarily due to the 
smaller bulk electron temperature (100 eV in the spectrum with hot electrons vs. 800 $\mathrm{eV}$ without) which leads to much larger radiative recombination rates (the magnitude of the decrease in recombination rates due to the $10 \%$ fraction of hot electrons that are unlikely to participate in recombination is dwarfed by the increase in recombination rates due to the small bulk temperature). The recombination feature in the spectrum with hot electrons is broadened because radiative recombination transitions from highly excited levels of Be- and L-like Ti become more likely as hot electrons spread out the charge state balance and increase the populations of excited levels.

\section{High-Energy Emission}

Measurements of high-energy emission from plasmas provide the least ambiguous evidence of high-energy electrons and also contain direct information about the functional form of the electron energy distribution. This is because free-free emission is more directly dependent on the electron distribution than the free-bound or boundbound processes already discussed, and the presence of high-energy electrons in a plasmas is a prerequisite for the emission of high-energy bremsstrahlung photons.

In Fig. 6, the high-energy (>10 keV) bremsstrahlung emission is orders of magnitude larger in the spectrum with hot electrons. In this figure, a Maxwellian distribution was used to model the hot electrons. Although hot electrons in this distribution have effects on $\mathrm{Ti}$ ionization balances and line emission that are very similar to the effects of the $10 \mathrm{keV}$ Gaussian distribution used in Fig. 3, the highenergy bremsstrahlung emission of such a narrow beam would be very different than that of the Maxwellian, as high-energy bremsstrahlung emission tends to mimic the functional form of the hot electron distribution [11]. Figure 6 shows that the absolute amplitude of the K-shell line emission is much larger in the presence of hot electrons. This effect can be particularly useful for hot electron diagnostics of plasmas with charge states dominated by $\mathrm{M}$ - or L-shell ions for which significant inner-shell emission is a clear indication of the presence of high-energy electrons [11].

\section{PRACTICAL CONSIDERATIONS}

In the first part of this paper, the effects of hot electrons on line emission and ionization balances were discussed. Such effects are dependent primarily on the amplification of collisional excitation and ionization rates and are most pronounced at small electron temperatures, when the bulk Maxwellian distribution has few electrons with sufficient energy to excite or ionize high-energy transitions and the rates due to the bulk electrons are correspondingly small. As the bulk temperature increases, more high-energy electrons are present in the bulk distribution, and only larger hot electrons fractions effectively compete with high-energy electrons in the bulk Maxwellian.

Figure 7 shows predicted and reported minimum hot electron fractions to which $\mathrm{K}$ and L-shell models of atoms with various nuclear charges are sensitive. The reported values of $f_{\min }$ are taken from the referenced sources, which use detailed collisionalradiative models. The predicted values of $f_{\min }$ are obtained using a simple coronal model with Z-scaled ionization potentials to predict the bulk electron temperature at 
which the K-shell (L-shell) ions are populated. Once that bulk temperature is known, the fraction of hot electrons with sufficient energy to affect the K-shell (L-shell) collisional excitation and ionization in the bulk Maxwellian is easily found and gives a baseline fraction of high-energy electrons below which the effects of hot electrons on line emission and ionization balance are difficult to detect.

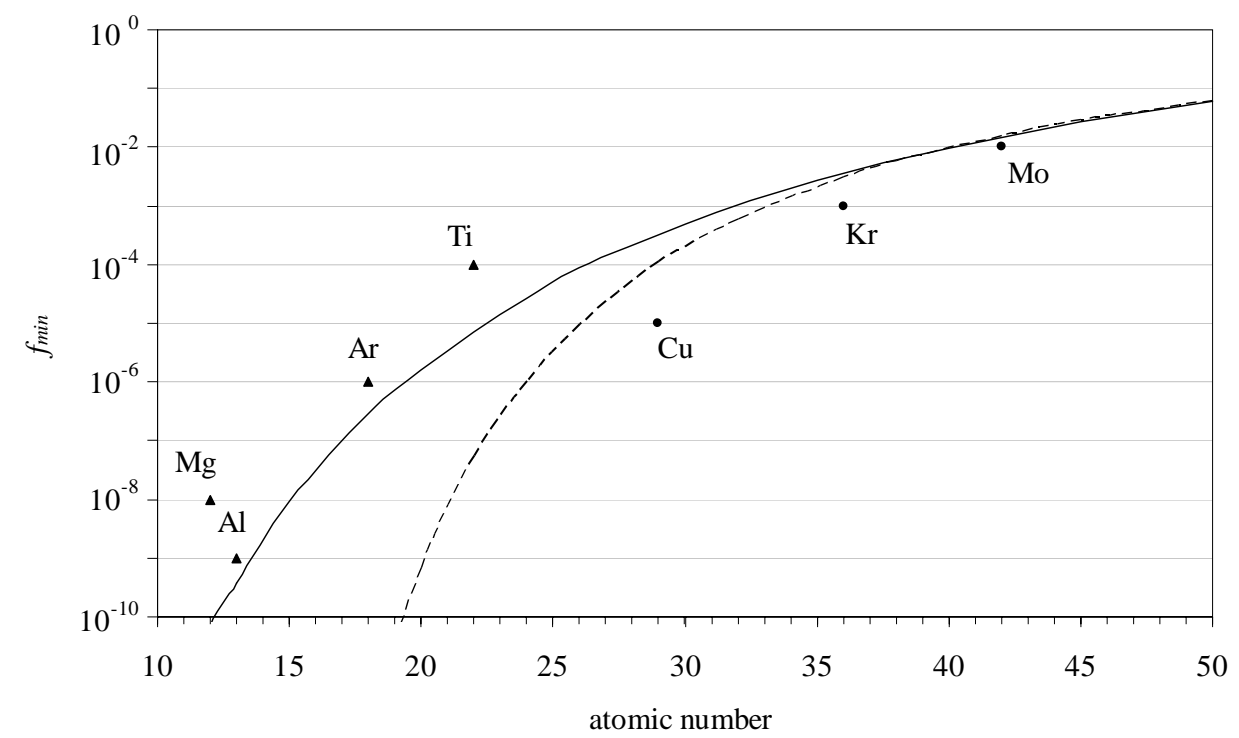

FIGURE 7. Predicted values of the minimum hot electron fraction $\left(f_{\min }\right)$ to which modeled K-shell (solid line) and L-shell (dashed line) spectra are sensitive. Reported sensitivity limits of various collisional-radiative models are given by triangles for K-shell Al [5], Mg [6], Ar [1], and $\mathrm{Ti}$ and by circles for L-shell $\mathrm{Cu}$ [3], $\mathrm{Kr}$ [2], and Mo [7].

Since the coronal temperature increases with increasing nuclear charges, line emission spectra from plasmas with low-Z ions are most sensitive to hot electrons. If hot electron diagnostics from line emission spectra are desired, low- $Z$ ions should be considered for the target material and the bulk temperature should be held as low as possible. Note that the sensitivity of hot electron diagnostics based on high-energy bremsstrahlung emission are not constrained in the same way by the bulk electron temperature. Since even high-temperature bulk Maxwellian electron distributions decay rapidly at high electron energies, high-energy bremsstrahlung emission can be used to determine arbitrarily small fractions of hot electrons as long as $\varepsilon_{H}$ and the energy range of the observed emission are sufficiently large.

\section{CONCLUSIONS}

Line and continuum emission from EUV to multi-keV spectral regions contain information about electron energy distributions. With sufficiently sophisticated measurements and modeling, hot electrons in plasma sources can be detected and measured and the functional form of the hot electron distribution can be characterized.

Hot electrons influence line emission by increasing and spreading out charge state balances compared to single-temperature plasmas, and by amplifying the intensities of high-energy lines, inner-shell satellite lines, and lines fed by radiative cascades in 
closed-shell ions. Hot electrons can also significantly alter the structure of low-energy inter-shell line transition features and radiative recombination features through their effects on excited level populations. Because most collisional rates are fairly insensitive to the modeled functional form and characteristic energy of the hot electron distribution as long as $\varepsilon_{H}>\Delta E$, line spectra can be a robust indicator of the presence and fraction of hot electrons in a plasma. Lower-Z elements are the best choices for experiments in which small fractions of hot electrons must be diagnosed from line spectra alone, since they require less energy in the bulk electron distribution to ionize and can therefore be more sensitive to $f$.

To determine more precisely the characteristics of the hot electron distribution, such as its characteristic energy and functional form, high-energy continuum and spectropolarimetry measurements can be used. Spectropolarimetry can be used to diagnose characteristic energies and anisotropy in hot electron distributions. Highenergy bremsstrahlung emission can help to determine the number of hot electrons and the general shape, characteristic energy, and energy cutoff of the distribution.

\section{ACKNOWLEDGMENTS}

The authors thank Kevin Fournier for helpful discussions and comments on this work. This work was supported by Lawrence Livermore National Laboratory under contract B520743. The work of S.H. was performed in part under the auspices of the U.S. Department of Energy by University of California Lawrence Livermore National Laboratory under contract No. W-7405-Eng-48.

\section{REFERENCES}

1. J. Abdallah, Jr., A.Ya. Faenov, I.Y. Skobelev, A.I. Magunov, T.A. Pikuz, T. Auguste, P. D’Oliveira, S. Hulin, and P. Monot, Phys. Rev. A 63, 032706 (2001).

2. S.B. Hansen, A.S. Shlyaptseva, and A.Y. Faenov et al., Phys. Rev. E 66, 046412 (2002).

3. K.B. Fournier, A.Ya. Faenov, and T.A. Pikuz, et al., Phys. Rev. E 67, 16402 (2003).

4. S.H. Glenzer, F.B. Rosmej, R.W. Lee, C.A. Back, K.G. Estabrook, B.J. MacGowan, T.D. Shepard, and R.E. Turner, Phys.Rev. Lett. 81, 365-368 (1998).

5. J. Abdallah, Jr., A.Ya. Faenov, D. Hammer, S.A. Pikuz, G. Csanak, and R.E.H. Clark, Physica Scripta 53, 705-711 (1996).

6. J. Abdallah, Jr., A.Ya. Faenov, T.A. Pikuz, M.D. Wilke, G.A. Kyrala, and R.E.H. Clark, J. Quant. Spectrosc. Radiat. Transf. 62, 1-11 (1999).

7. A.S. Shlyaptseva, S.B. Hansen, V.L. Kantsyrev, D.A. Fedin, N. Ouart, K.B. Fournier, and U.I. Safronova, Phys. Rev. E 67, 026409 (2003).

8. T. Kato, T. Fujiwara, and Y. Hanaoka, Astrophysical Journal 492, 822-832 (1998)

9. U. Feldman, Physica Scripta 65, 185-192 (2002).

10. F.B. Rosmej, J. Phys. B 30, L819-L828 (1997).

11. S.B. Hansen and A.S. Shlyaptseva (submitted to Phys. Rev. E 2004).

12. M. F. Gu, Astrophysical Journal 582, 1241-1250 (2003).

13. P. Beiersdorfer, G. Brown, S. Utter, P. Neill, K. J. Reed, A. J. Smith, and R. S. Thoe, Phys. Rev. A 60, 4156-4159 (1999).

14. A.S. Shlyaptseva, V.L. Kantsyrev, B.S. Bauer, P. Neill, C.Harris, D.A. Fedin, S. Hansen, N. Ouart, P. Beiersdorfer, A.G. Petrashen, and U. I. Safronova, University of California Report No. UCRLJC-146907, 339-358 (2001). 